\title{
Cancer Radiotherapy Based on Attenuation Coefficient
}

\author{
Bibek Koirala ${ }^{1,3}$, Saddam Husain Dhobi ${ }^{1,2,3^{*}}$, Kishori Yadav ${ }^{1,3}$, Jeevan Joyti Nakarmi ${ }^{1,3}$, Khem Poudyal ${ }^{4}$ \\ ${ }^{1}$ Department of Physics, Patan Multiple Campus, Tribhuvan University, Lalitpur-44700, Nepal \\ ${ }^{2}$ Robotics Academy of Nepal, Lalitpur-44700, Nepal \\ ${ }^{3}$ Innovative Ghar Nepal, Lalitpur-44700 \\ ${ }^{4}$ Institute of Engineering, Pulchowk Campus, Tribhuvan University, Lalitpur-44700, Nepal \\ *Corresponding Author
}

\begin{abstract}
The objective of this work is to select the best chemomaterial based on the attenuation coefficient. WinXcom software is used to study the attenuation coefficient of tissue, bone, gold, copper oxygen, water, and mixture. The mixture contains 0.5 fractional weight of gold, 0.3 fractional weight of copper, and 0.2 fractional weight of oxygen. The attenuation coefficient determines the loss of radiation when entering into the medium and loss determines the penetration depth of radiation and damage of materials. The amount of radiation loss when the radiation passes through samples gives the information to select the best chemotherapy materials. Because loss of radiation may be due to absorption, scattering ad emission of electrons from the target. In this work, we consider loss due to the emission of the electron because emitted electrons are used to kill the tumor cell. In this work, the mixture of gold, copper, and oxygen sample has a medium attenuation coefficient that means the emitted electron has the best preformation to kill the cancer cell. This is because the emitted electron has not had enough energy to reach healthy tissue and bone. Therefore, both healthy tissue and bone become safer when the mixture sample is used as a chemo-material instead of a single element. If a single element $(\mathrm{Au}, \mathrm{Cu}$, and $\mathrm{O})$ is used as chemo-material for cancer therapy, the energy of electron emitted by Au is greater and it may affect the tissue and bone while the energy emitted from $\mathrm{Cu}$ and $\mathrm{O}$ has less energy and can't kill the larger amount of cancer cell. Therefore, if the mixture of chemo-material of gold, copper, and oxygen is better and safer than the individual element to load the tumor for radiotherapy.
\end{abstract}

Keywords: Attenuation coefficient, tissue, bone, gold, tumor cell, penetration, etc.

\section{INTRODUCTION}

A cell is the basic building block of all living things and normally grows and divides (multiply) to replace old cells. A cell becomes cancerous when it grows quickly and uncontrollably. Malignant tumors are cancer, can spread to other tissues and organs near the tumor. Literature shows all ages people get cancer but older than 55year is more common. The time from first cell change to time cancer is detected is the latency period. The chemical that causes cancer are benzene, beryllium, asbestos, vinyl chloride, and arsenic are known human carcinogens. Chloroform, DDT, formaldehyde, and polychlorinated biphenyls (PCBs) are examples of possible human carcinogens [1].
Malignant tumors are mostly treated by external beam radiotherapy, possibly combined with chemotherapy. External radiotherapy and radionuclide therapy both use ionizing radiation to damage the DNA of cancerous cells [2-3]. Chemotherapy treatments are used to inhibit vigorously growing malignant cells with anticancer agents, in general, it gives cyclic manners and it is the traditional method [4]. Anticancer drugs are used to destroy the specific cancer cell but also affect the normal cell in circumstances. Each treatment has its specific side effects and treatment strategy is also different [5]. In this, more than one type of therapy is adopted at a time to treat cancer, such as radiation therapy, surgery, and/ or hyperthermia [6].

Metals and metal compounds are used in medicine for several thousands of years. Literature shows that anti-cancer activities most active metals as arsenic, antimony, bismuth, gold, vanadium, iron, rhodium, titanium, gallium, platinum and gold (I) and (III) compounds show anti-tumor activities and have a high potential to treat cancer cells or tumors [7]. According to WHO report 2005, more than 11 million cases of cancer are diagnosed each year and expected 16 million by 2020. Most treatments are based on antineoplastic chemotherapy and about 100 different antineoplastic drugs are used nowadays [8]. Two theories provide a rationale for dose intensification, the Goldie-Coldman hypothesis, and the Norton-Simon hypothesis. Chemotherapy shrinks the tumor and an increase in the repetition of therapy shows that shrink is more effective in a short time. Anthracycline and taxane chemotherapy are primary recurrence and breast cancer mortality [9].

Radiotherapy is also known as radio ionization used to kill the tumor cell by energetic electron produced by hitting the target. The phenomena of production such energetic electrons are the photoelectric effect, Compton effect, and Auger electrons. The Auger electrons have typically low energy and would travel short distances but damage DNA when emitting near the nucleus. The application of gold and other inorganic radiosensitizers in combination with chemical effects are discussed by Hainfeld et al. (2008), Rashid et al. (2018), and Rashida et al. (2019). 


\section{LITERATURE REVIEW}

Randomized trials have shown that radiotherapy, chemotherapy, and endocrine therapy for early-stage breast cancer can reduce the rates of recurrence and breast cancer death. But have shown that radiotherapy and chemotherapy can increase the risk of heart disease [10-11]. Gold is an excellent absorber of X-rays and when tumors are loaded with gold. A high dose of X-ray can be used on the cancerous tissue compared with the dose received by normal tissue during radiotherapy treatment. Gold nanoparticles have shown therapeutic efficacy in animal trials and these results are reviewed [12].

Herold et al (2000) injected 1.5-3.0 $\mu \mathrm{m}$ gold particles directly into a tumor and irradiated high-energy photons on it. A similar, experiment was carried out at the European Synchrotron Radiation Facility with 50keV energy photon on rat F98 brain gliomas by loaded tumor with $1 \%$ iodine. When $\mathrm{X}$-rays impinge on the matter, several processes can result such as scattering of photons, photoelectrons, Compton electrons, Auger electrons, and fluorescence photons. When an incident photon ejects an electron from an inner shell of an atom. The photoelectron acquires the kinetic energy of the primary beam minus its binding energy, and this kinetic energy determines the range of electrons in the tissue. If the phenomena emit low energy electron penetration is low but if phenomena emit high energy electrons then penetration is high.

Oral cancer is one of the most commonly diagnosed malignancies in the head and neck region and currently choices are surgery, radiotherapy, and/or chemotherapy. Radiotherapy is a critical therapy for cancer patients that causes DNA damage directly via ionizing radiation (IR) or by indirectly generating oxidative damage via reactive oxygen species (ROS), thereby leading to cancer cell destruction. Therefore in this work authors are interested in radiotherapy which depends upon the energy of the photon, material, or element selection to be loaded in the tumor, the thickness of skin, size of the tumor, etc. Cancer stem cells considered the seeds of cancer have been confirmed to be in a quiescent state in most established tumors, with their innate radio resistance helping them survive more easily when exposed to radiation than differentiated cancer cells [13].

Radiotherapy plays a central part in curing cancer about $50 \%$ of cancer patients use radiotherapy and to manage $40 \%$ of patients who are cured. The improving outcomes from radiotherapy focussed almost entirely on the cancer cell itself by ignoring complex biological interactions between the tumors and the stroma in which it grows called tumor microenvironment (TME) [14].

Radiotherapy is one of the best therapy among three cancer therapy, together with surgery and chemotherapy. The effect of therapeutic has been considered mainly in the local region with direct or indirect DNA damage of irradiated cancer cells. The potential of radiotherapy used with immunotherapy to foster systemic tumor regression in advanced stage cancers with distant metastases has opened a new field of investigation into the mechanisms of the underlying immune response induced by radiotherapy [15].

\section{Theory and formulation}

Let us consider, when a photon beam passes through an absorber, the photon is attenuated. The attenuation depends on the scattering and various absorption processes. According to Lambert-Beer law, $I=I_{0} e^{-\mu x}$

Here, $I_{0}$ is the intensity of incident photon measured without entering the photon in the sample, I is the intensity of transmitted photon measure after passing the photon through the sample, $x$ is the thickness of sample and $\mu$ is absorption coefficient. The mass attenuation coefficient $\left(\frac{\mu}{\rho}\right)$ with experiential data is given for the elements [16] as

$$
\frac{\mu}{\rho}=\frac{1}{\rho x} \ln \left(\frac{I_{0}}{I}\right)
$$

Here, $\rho$ is density of material $\left(\mathrm{g} / \mathrm{cm}^{3}\right), \mu / \rho\left(\mathrm{cm}^{2} / \mathrm{g}\right)$ theoretical mass attenuation coefficient. In general $\mu / \rho$ for any chemical compound or mixture of elements is given by mixture rule [15], $\left(\frac{\mu}{\rho}\right)=\sum_{i} w_{i}\left(\frac{\mu}{\rho}\right)_{i}$

Here, where $w_{i}$ is the weight fraction and $\left(\frac{\mu}{\rho}\right)_{i}$ is mass attenuation coefficient of the $i^{\text {th }}$ constituent element. $\left(\frac{\mu}{\rho}\right)$ does not depend on the particular phase (gas, liquid, or solid) of the material. The fractional weight $\left(w_{i}\right)$ for a chemical compound is given by $w_{i}=\frac{n_{i} A_{i}}{\sum_{i} n_{i} A_{i}}$

The mass attenuation coefficient of considering material and samples in this work is calculated by the WinXCom program. This program also gives information of partial cross sections for incoherent and coherent scattering, photoelectric absorption, and pair production at energies from $1 \mathrm{keV}$ to 100 $\mathrm{GeV}$ but in this work, only $0.001 \mathrm{MeV}$ to $0.2 \mathrm{MeV}$ are used [17-19].

The total molecular cross-section is defined as

$$
\sigma_{t, m}=\left(\frac{\mu}{\rho}\right) \frac{M}{N_{A}}
$$

Here, $M$ is the molecular weight, $N_{A}$ is Avogadro's number and similarly total atomic cross-section $\left(\sigma_{t, a}\right)$ is define $\operatorname{as} \sigma_{t, a}=\frac{1}{N_{A}} \sum_{i} f_{i} A_{i}\left(\frac{\mu}{\rho}\right)_{i}=\frac{\sigma_{t, m}}{\sum_{i} n_{i}}$

Here, $f_{i}$ is the fractional abundance of element $i$ concerning the number of atoms, $n_{i}$ is the number of formula units, $A_{i}$ is the atomic weight of the constituent element $i$. Also the total electronic cross-section $\left(\sigma_{t, e l}\right)$ for the individual element is defined as [20], $\sigma_{t, e l}=\frac{1}{N_{A}} \sum_{i} \frac{f_{i} A_{i}}{Z_{i}}\left(\frac{\mu}{\rho}\right)_{i}=\frac{\sigma_{t, a}}{Z_{e f f}}$ 
The ratio of total atomic to electronic cross-sections define effective atomic number $\left(Z_{\text {eff }}\right)$ as $Z_{e f f}=\frac{\sigma_{t, a}}{\sigma_{t, e l}}$

\section{RESULTS AND DISCUSSION}

The mass attenuation coefficient for tissue (soft tissue) is shown below in Figure 1. The nature shows that with increasing the energy of photon the mass attenuation coefficient decrease. The mass attenuation is high at lowenergy photons and low at high-energy photons. The nature of the graph for both mass attenuation coefficient and massenergy attenuation coefficient is either decreasing in general [20]. The mass and energy attenuation was measured using the WinXcom program with element, mixture, and compound formula.

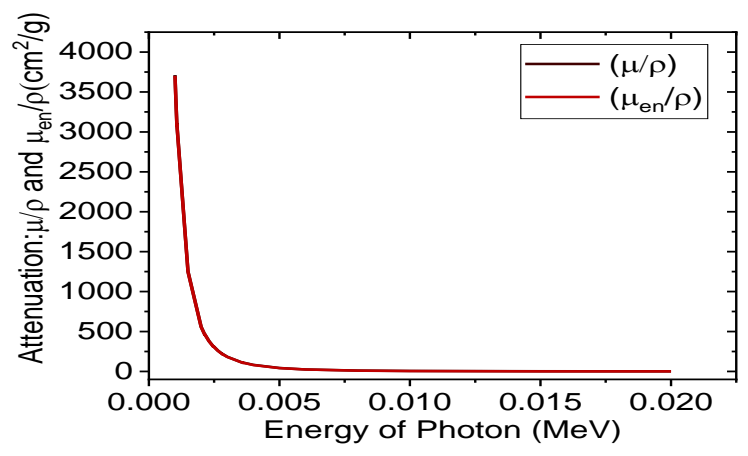

Figure 1: Attenuation of Soft tissue (ICRU-44) with photon energy

The mass and mass-energy attenuation coefficient of bone have similar nature with the same as tissue. But in between $0.001 \mathrm{MeV}$ to $0.005 \mathrm{MeV}$ two peaks are observed for both mass attenuation and mass-energy attenuation coefficient for bone.

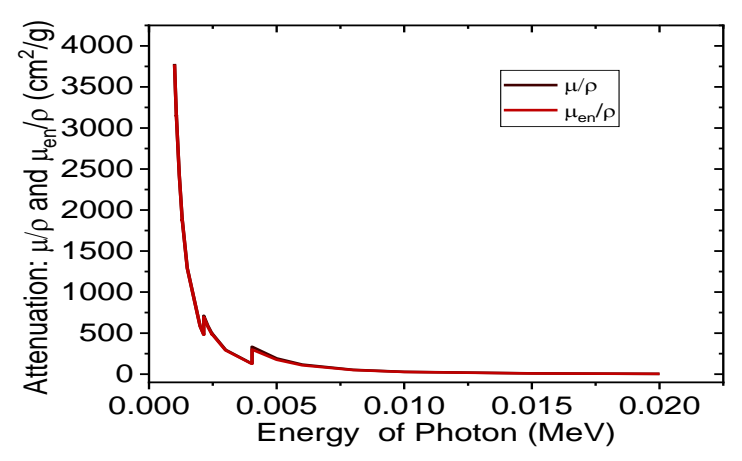

Figure 2: Attenuation Bone Cortical (ICRU-44) with photon energy

Figure 1 and Figure 2 represent show the mass attenuation and mass-energy attenuation coefficient nature with incidence energy of the photon. When the energy of $0.001 \mathrm{MeV}$ to $0.2 \mathrm{MeV}$ incidence on the normal soft tissue and bone cortical. The larger value of the attenuation coefficient indicates the incidence beam is quickly weakened (attenuated) when passes through the medium while the small value shows the incidence photon is easily transparent to the medium. The attenuation for tissue and bone is high as shown in Figures 1 and 2 which means the tissue and bone, weak the energy of photon and photon can't travel a longer distance. This means photon is highly absorbed by soft tissue and bone.

Figure 3 represent the mass attenuation coefficient of gold, copper, and oxygen which are the material loaded to the tumor for radiotherapy. When the tumor is loaded with either gold, copper, and oxygen and $0.001 \mathrm{MeV}$ to $0.2 \mathrm{MeV}$ energy photon incidence on the gold, copper, and oxygen atoms. The atoms are ionized and emit electrons and these electrons carry the remaining energy of photon which is used to damage the tumor cell. On comparing the mass attenuation of gold, copper, and oxygen the mass attenuation of gold is higher than copper and oxygen. This means the photon is attenuated highly in the gold medium than others and travels less distance. But when passes through the copper and oxygen medium photon go less attenuated and travel a large distance.

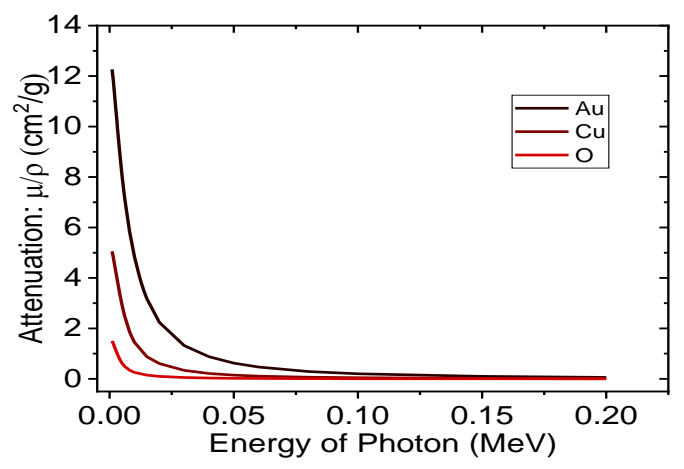

Figure 3: Attenuation of Gold, Copper, and Oxygen with photon energy

Figure 4 shows the mass attenuation of water, the mass attenuation of water is very low that means the incidence of photon travel or penetrate long distances in water medium. The water mass attenuation is less than all other elements consider in this work (gold, oxygen, copper, tissue, and bone). Therefore, for the same thickness, the penetration of the same energy photon is high for water and lower than tissue and bone.

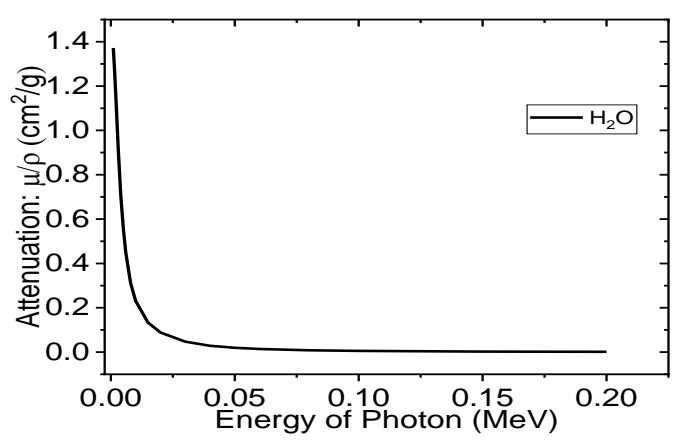

Figure 4: Attenuation of Liquid water with photon energy 
Figure 5 shows the variation of mass attenuation of gold, copper, oxygen, and mixture ( $\mathrm{Au}: \mathrm{Cu}: \mathrm{O}$ is $0.5: 0.3: 0.2$ by fraction weight). The mass attenuation of gold is higher than other consider samples in this work. When the same energy, the same thickness of material received the incidence photon then in the gold medium the penetration of photon is lower than other. If the solution or the mixture is loaded in tumor and photon of energy $(0.001 \mathrm{MeV}$ to $0.2 \mathrm{MeV})$ incidence on it. The incidence photon energy causes ionization of loaded particles in tumor cells because the energy of incidence photon is higher ( $\mathrm{MeV}$ range) than threshold energy $(\mathrm{eV})$ of mixture or individual. The ionization of the atom (photoelectric effect) is taking place and the remaining energy of the photon is carried by ejected or ionized electron. This ejected electron may affect the DNA of the healthy cell but due to less energy of the ejected electrons from the loaded solutions in the tumor. Since the energy of an ejected photon is less, therefore, can't travel long distance and hence only damage tumor cell and reduce the size of the tumor safely.

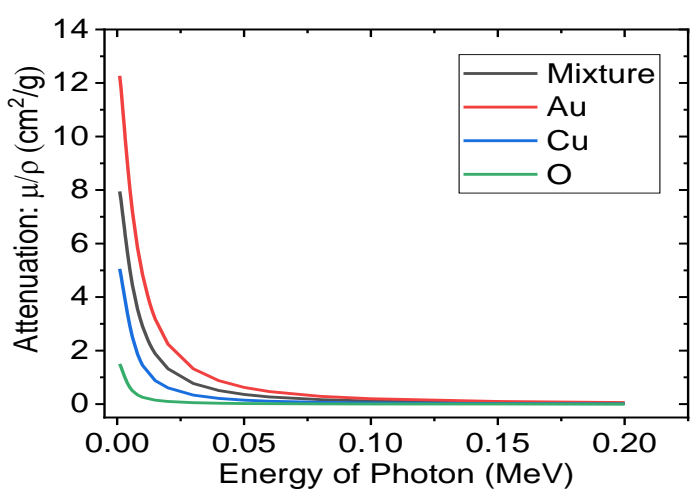

Figure 5: Variation of Attenuation with photon energy

\section{CONCLUSION}

The ejected electron after the incidence of photon on solution loaded in tumor cell has different penetration to the tumor and healthy cell. The energy of ejected electron penetration depends upon the energy of the incidence photon. The energy of the ejected electron is less than the penetration of electron is less and hence guaranteed to kill the tumor cell only but if energy is higher the penetration is high and may damage the healthy cell or DNA of the cell. This shows that radiotherapy depends on several factors like the energy of the photon, material loaded in the tumor, the thickness of the tumor as well as skin, etc. Among the selective metal and mixture, gold's high attenuation coefficient is greater than others, which means when photon incidence on loaded gold and ejected electron has high. The ejected electron due to high energy may damage the healthy cell therefore mixture sample of gold, copper, and oxygen is best. This is because the attenuation coefficient of the individual is either low or high, lower attenuation has low intensity to kill tumor cells or high has greater change to damage the healthy

\section{REFERENCE}

[1] Agency for Toxic Substances and Disease Registry: Division of Health Assessment and Consultation, "Chemicals, Cancer, and You," 2009,

https://www.atsdr.cdc.gov/emes/public/docs/Chemicals, \%20Cance r,\%20and\%20You\%20FS.pdf acessed at $5^{\text {th }}$ december, 2021.

[2] C. Washington, D. Leaver, Principles and Practice of Radiation Therapy, Elsevier, Amsterdam 2015, pp.12-16.

[3] T. W. Speer, Targeted Radionuclide Therapy, Lippincott Williams \& Wilkins, PA 2010, pp. 145-150.

[4] T. Priestman, Cancer Chemotherapy in Clinical Practice, SpringerVerlag London 2012, p.1-16.

[5] A. Alam, U. Farooq, R. Singh, V.P. Dubey, S. Kumar, R. Kumari, K. K. Naik, B.D. Tripathi and K.L. Dhar, Chemotherapy Treatment and Strategy Schemes: A Review, Open Access Journal of Toxicology, 2(5), 2018, pp.1-2.

[6] R. Adam, G. Pascal, D. Castaing, D. Azoulay, V. Delvart, et al., Tumor progression while on chemotherapy: a contraindication to liver resection for multiple colorectal metastases?, Annals of Surgery, 240(6), 2004, pp.1052- 1061.

[7] B. Desoize, Metals and Metal Compounds in Cancer Treatment, Anticancer Research, 24, 2004, pp.1529-1544.

[8] Pan American Health Organization and WHO America, Safe Handling of Hazardous Chemotherapy Drugs In Limited-Resource Setting, Washington, D.C. 2013, pp.1-62.

[9] S. A. Hurvitz, Dose intensification of chemotherapy for early breast cancer in the age of de-escalation, The Lancent, 393, 2019, pp.1390-1392.

[10] Early Breast Cancer Trialists' Collaborative Group, Effect of radiotherapy after breast-conserving surgery on 10 -year recurrence and 15-year breast cancer death: meta-analysis of individual patient data for 10801 women in 17 randomised trials, Lancet, 378, 2011, 1707-1716.

[11] A. Matthews, S. Stanway, R.E. Farmer, Long term adjuvant endocrine therapy and risk of cardiovascular disease in female breast cancer survivors: systematic review, BMJ, 363, 2018, pp.3845-3850.

[12] J. F. Hainfeld, F. A. Dilmanian, D. N. Slatkin and H. M. Smilowitz, Radiotherapy enhancement with gold nanoparticles, Journal of Pharmacy and Pharmacology, 60, 2008, pp.977-985.

[13] Y. Liu, M. Yang, J. Luo and H. Zhou1, Radiotherapy targeting cancer stem cells "awakens" them to induce tumour relapse and metastasis in oral cancer, International Journal of Oral Science 12, 2020, pp.12-14.

[14] H. E. Barker, J.T.E. Paget, A.A. Khan, and K. J. Harrington, The Tumour Microenvironment after Radiotherapy: Mechanisms of Resistance and Recurrence, Nature Review Cancer, 15(7), 2015, pp.409-425.

[15] H. Sato, S. Demaria and T. Ohno, The role of radiotherapy in the age of mmunotherapy, Japanese Journal of Clinical Oncology, 51(4), 2021, 513-522.

[16] D. F. Jackson and D. J. Hawkes, X-ray attenuation coefficients of elements and mixtures, Physics Reports, 70 (3), 1981, pp.169-233.

[17] M. J. Berger and J. H. Hubbell, XCOM: Photon Cross Sections Database: Web Version 1.2, National Institute of Standards and Technology, Gaithersburg, Md, USA, 1987.

[18] L. Gerward, N. Guilbert, K. B. Jensen, and H. Levring, WinXCom: a program for calculating X-ray attenuation coefficients, Radiation Physics and Chemistry, 71 (3-4), 2004, pp.653-654.

[19] K. Singh and L. Gerward, Summary of existing information on gamma-ray and X-ray attenuation coefficients of solutions, Indian Journal of Pure and Applied Physics, 40(9), 2002, pp.643-649.

[20] S. R. Manohara, S. M. Hanagodimath, and L. Gerward, Energy dependence of effective atomic numbers for photon energy absorption and photon interaction: studies of some biological molecules in the energy range $1 \mathrm{keV}-20 \mathrm{MeV}$, Medical Physics, $35(1), 2008$, pp.388-402. 